\title{
Online Social Chat Application
}

\author{
Mrs. Hadke Seema ${ }^{1}$, Karad Prajakta ${ }^{2}, K_{\text {Komal Raina }}{ }^{3}$, Raskar Shital $^{4}$, Valvi Aboli ${ }^{5}$ \\ Asst Prof, IT, Bharti Vidyapeeth College of Engineering for Women, Pune, India ${ }^{1}$ \\ BE, IT, Bharti Vidyapeeth College of Engineering for Women, Pune, India ${ }^{2,3,4,5}$
}

\begin{abstract}
Social media could be a nice medium for exploring developments that matter most to a broad audience and it's the suggests that of interactions among individuals during which they produce, share, and exchange info and ideas in virtual communities and networks. Social media technologies defy many alternative forms together with magazines, net forums, weblogs, social blogs, small blogging, wiki, social network, podcasts, pictures or photos, video, rating and social bookmarking. Social networking websites like facebook, gmail even have giant numbers of users wherever individuals chat with one another and share their views and issues over varied things. Social chat through mobile is additionally effective means for communication.
\end{abstract}

Keywords: Online social network; Misinformation; Disinformation; Cognitive Psychology.

\section{INTRODUCTION}

The recently rising quality of on-line social network sites, like Facebook, Twitter and Google+, has created an enormous impact on the landscape of knowledge sharing and discovery today. owing to the advantage of scattering data to wider audiences, such OSNs change their users to browse and create new content at an equivalent time, so shifting the role of knowledge broker from some dedicated news sources to a a lot of various cluster of people. whereas this advantage permits on-line users a lot of rooms to decide onthat content to follow, it additionally makes OSNs fertile grounds for the wide unfold of misinformation which may cause undesirable consequences.

Internet could be a nice supply of knowledge. it's additionally referred to as the net of deception. the employment of communication channels of the web to propagate false data has become quite common. the appearance of social networks has created each user a self-publisher with no written material, checking for factual accuracy and clearly with no responsibility. The facts square measure bestowed with no authority and for legion users seeing them on their monitor is itself a certificate of honesty of knowledge being bestowed to them.

\section{RELATED WORK}

Unlike other steganography approaches, studies on data hiding in short message service (SMS) are quite limited. The first investigation on SMS steganography was reported by Shirali-Shahreza [8], which utilized image as cover media and SMS as carrier to transfer the hidden message to the recipient.

In this method, a black and white (B\&W) image is used to transfer the hidden message by changing the intensity of pixels.

However, low capacity ( 27 bytes) is the main drawbackof the mentioned technique, due to the use of only black and white image rather than using full colour.

A few attempts on this line of work have been proposed in the literature. In [7], Shah et al. study the problem of finding the sources of rumors on the SIR model using a maximum likelihood estimator.

They extend their solution to general graphs via finding Breath First Search (BFS) trees at different nodes. Since BFS tree is not unique, this methods might not be consistent especially when multiple sources exist.

Lappas et al proposed the $\mathrm{k}$-Effector problem which tries to find a set of at most $\mathrm{k}$ nodes that best explain the current activationstate of the network. They suggest an optimal algorithm for this problem on trees, however, the extension of that algorithm does not appear to work well on general graphs.

InQazvinian et al. propose a framework to detect people that endorse and further spread rumors out to a wider population. This method, however, requires a large amount of real training data for its proper function. 


\section{ARCHITECTURE DIAGRAM}

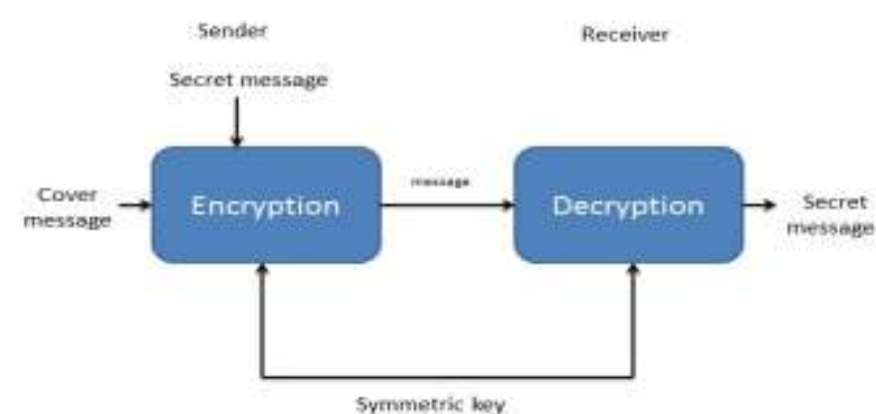

Encryption- decryption concept in social chat application

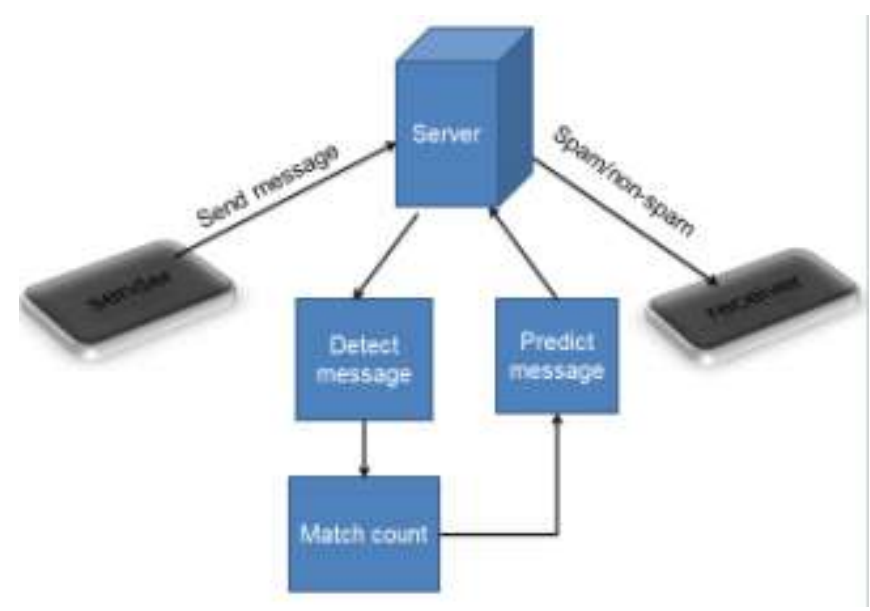

Architectural view

\section{IMPLEMENTATION}

In first stage, home page is displayed as per shown in fig 1

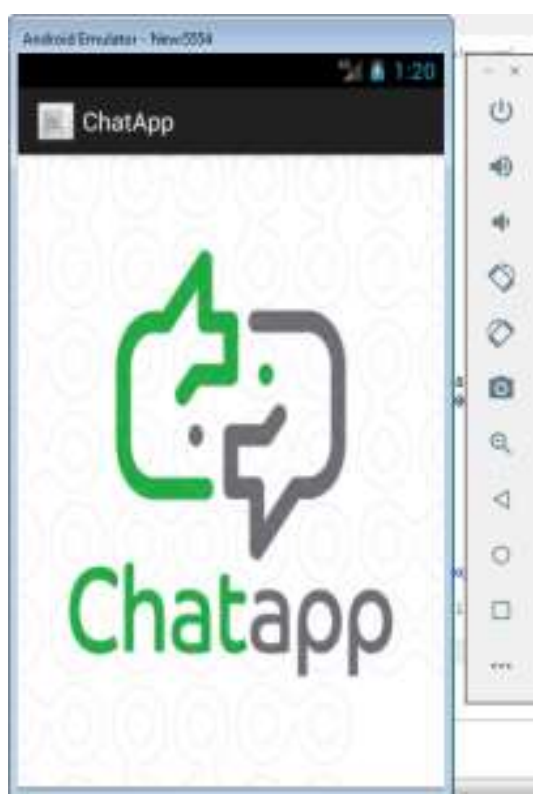

Fig 1

First module is login module of user. User will login to application by entering email-id and mobile number as shown in fig 2. 


\section{IJARCCE}

International Journal of Advanced Research in Computer and Communication Engineering ISO 3297:2007 Certified

Vol. 6, Issue 4, April 2017

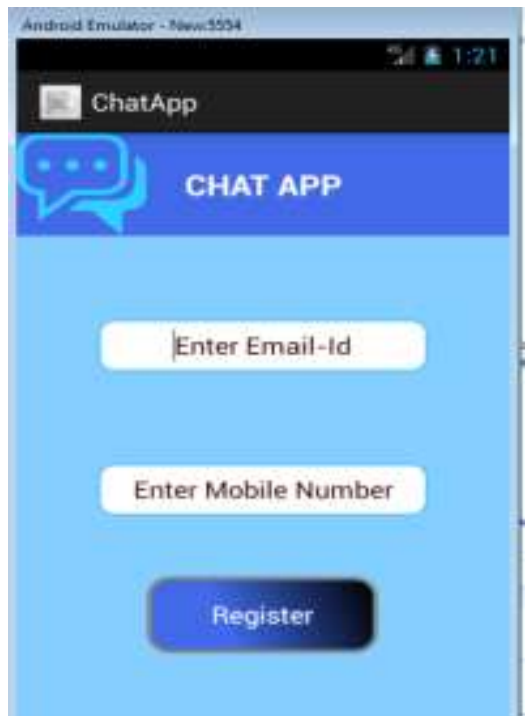

Fig 2

After login, OTP is generated. If OTP is correct next login page will be displayed. If we enter wrong OTP then message will displayed as wrong OTP as shown in fig 3(a), 3(b), 3(c).

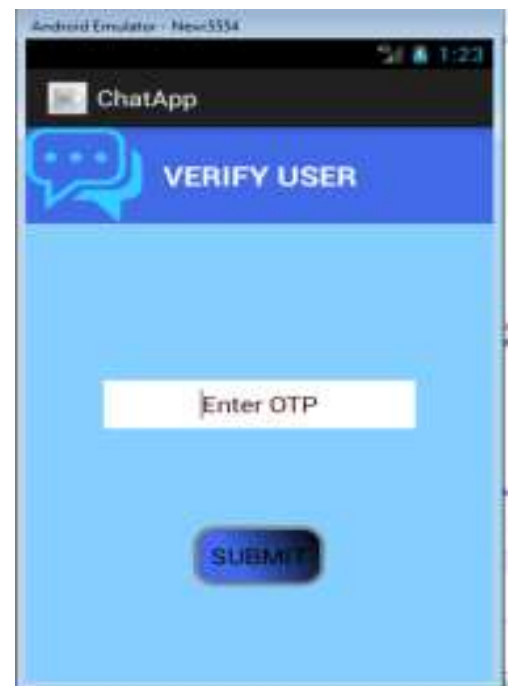

Fig. 3(a)

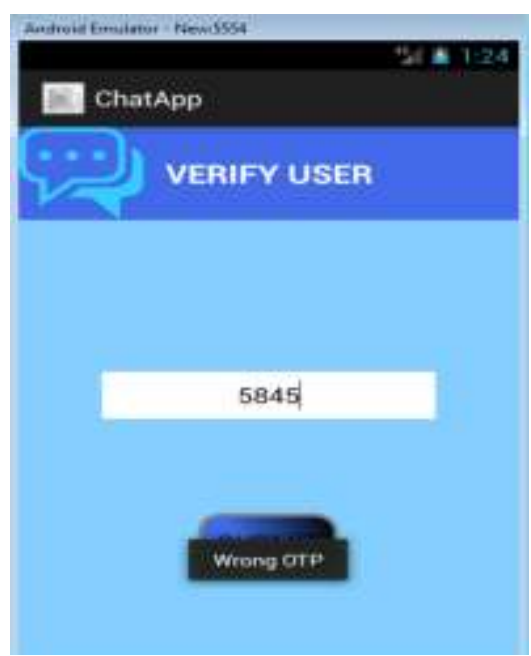

Fig 3(b) 


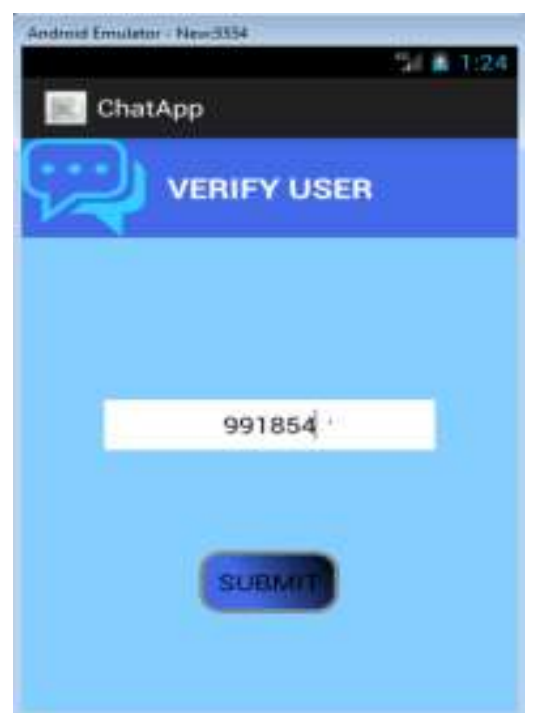

Fig 3(c)

In profile, Message can be send to various friends as mentioned in list. This is shown in fig 4.

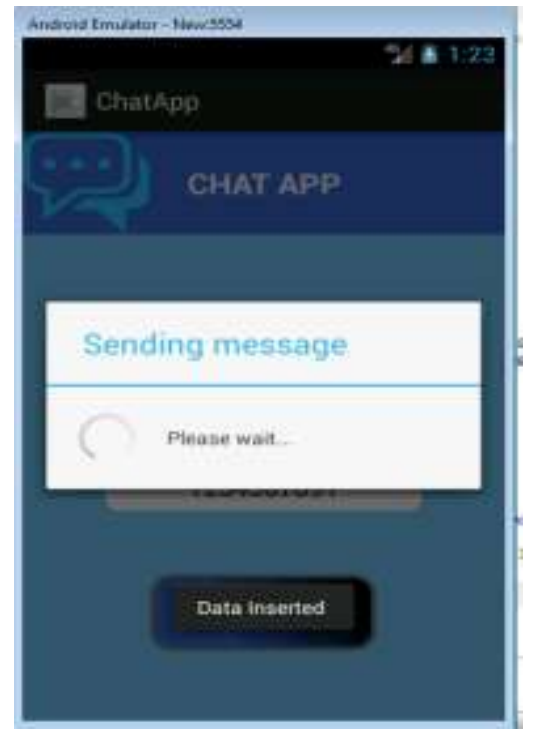

In user profile, chat list and contacts are displayed shown in fig .5(a)and 5(b).

\section{CONCLUSION}

We will develop a chat application which provides function like strong securities as well as removing spam/unwanted messages.

\section{REFERENCES}

1. M. Shirali-shahreza, "stealth steganography in sms", proceedings of The third ieee and ifip international conference on wireless and optical Communications networks (wocn), april, 2006.

2. M. Shirali-shahreza, and m. H. Shirali-shahreza, " text steganography In sms", international conference on convergence information Technology, pp. 2260-2265, 2007.

3. K. F. Rafat, "enhanced text steganography in sms", international Conference on computer, control and communication, pp. 1-6, 2009.

4. M. H. Shirali-shahreza, and m. Shirali-shahreza, "sending mobile Software activation code by sms using steganography", third International conference on intelligent information hiding and Multimedia signal processing, 1, pp. 554-557, 2007.

5. M. H. Shirali-shahreza, and m. Shirali-shahreza, "steganography in Sms by sudoku puzzle", international conference on computer systems And applications, pp. 844-847, 2008.

6. J. Singh, R. Ruhl, and D. Lindskog," Secure GSM OTA SIM cloning attack and cloning resistance in EAP- SIM and USIM ",International Conference on Social Computing(SocialCom), pp. 10051010, 2013.

7. Lewandowsky S, Ecker UK, Seifert CM, Schwarz N, Cook J (2012) Misinformation and its correction continued influence and successful debiasing. PsycholSci Public Interest 13(3):106131. 